\title{
Diagnosing Plasma Gradients Using Spectral Line Shapes
}

C. A. Back, I. Golovkin, R. Mancini, T. Missalla, O. L. Landen, R. W. Lee, L. Klein

This article was submitted to $15^{\text {th }}$ International Conference on Spectral Line Shapes Berlin, Germany July $10-14,2000$

U.S. Department of Energy

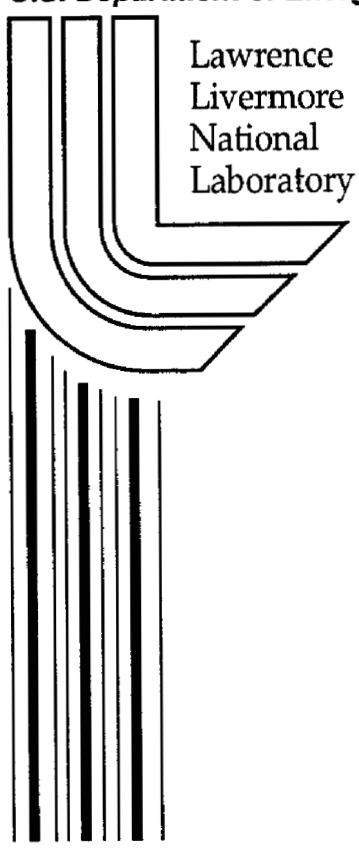

November 13, 2000 


\section{DISCLAIMER}

This document was prepared as an account of work sponsored by an agency of the United States Government. Neither the United States Government nor the University of California nor any of their employees, makes any warranty, express or implied, or assumes any legal liability or responsibility for the accuracy, completeness, or usefulness of any information, apparatus, product, or process disclosed, or represents that its use would not infringe privately owned rights. Reference herein to any specific commercial product, process, or service by trade name, trademark, manufacturer, or otherwise, does not necessarily constitute or imply its endorsement, recommendation, or favoring by the United States Government or the University of California. The views and opinions of authors expressed herein do not necessarily state or reflect those of the United States Government or the University of California, and shall not be used for advertising or product endorsement purposes.

This is a preprint of a paper intended for publication in a journal or proceedings. Since changes may be made before publication, this preprint is made available with the understanding that it will not be cited or reproduced without the permission of the author.

This report has been reproduced directly from the best available copy.

Available electronically at http://www.doc.gov/bridge

Available for a processing fee to U.S. Department of Energy

And its contractors in paper from

U.S. Department of Energy

Office of Scientific and Technical Information

P.O. Box 62

Oak Ridge, TN 37831-0062

Telephone: (865) 576-8401

Facsimile: (865) 576-5728

E-mail: reports@adonis.osti.gov

Available for the sale to the public from

U.S. Department of Commerce

National Technical Information Service

5285 Port Royal Road

Springfield, VA 22161

Telephone: (800) 553-6847

Facsimile: (703) 605-6900

E-mail: orders@ntis.fedworld.gov

Online ordering: http:/ / www.ntis.gov/ordering.htm

\section{OR}

Lawrence Livermore National Laboratory

Technical Information Department's Digital Library

http: / / www.llnl.gov/tid/Library.html 


\title{
Diagnosing Plasma Gradients Using Spectral Line Shapes
}

\author{
C. A. Back, I. Golovkin ${ }^{\text {a }}$, R. Mancini ${ }^{\text {a }}$, T. Missalla, O. L. Landen, \\ R. W. Lee, and L. Klein ${ }^{b}$
}

\author{
Lawrence Livermore National Laboratory, L-2I, P.O. Box 808, Livermore, CA 94551 \\ ${ }^{a}$ University of Nevada Reno, Dept. of Physics, Reno, NV 89557 \\ ${ }^{b}$ Howard University, Dept. of Physics and Astronomy, Washington, D.C. 20059
}

\begin{abstract}
The development of a set of stable implosions using indirectly driven plastic microspheres with argon $(0.1 \mathrm{~atm})$ doped deuterium $(50 \mathrm{~atm})$ has provided a unique source for testing the plasma spectroscopy of the high energy density imploded core. The core reaches electron densities of $>10^{2+} \mathrm{cm}^{-3}$ with temperatures of $-1 \mathrm{keV}$ and has been shown to be reproducible on a shot to shot basis. Moreover, it has been shown that not only the peak temperature and density are consistent, but that the temporal evolution of the mean temperature and density of the final phase of the implosion is also reproducible.

These imploding cores provide a unique opportunity to test aspects of plasma spectroscopy that are difficult to study in other plasmas and to develop methods to test stable hydrodynamics. We will present experimental results and discuss spectroscopic analysis algorithms to determine consistent temperature and density fits to determine gradients in the plasma.
\end{abstract}

\section{INTRODUCTION}

In hot dense plasmas created in high-powered laser experiments, spectroscopy has been a very powerful tool for diagnosing the plasmas. In the simplest application, the mere presence of line emission is used to identify ion species. The electron temperature or density may be determined by techniques such as measuring the ratio of different spectral lines if a suitable plasma kinetics model can be constructed. Or using line broadening theories, electron densities or ionic temperatures can be deduced by analyzing line widths. With the ability to use more complex atomic models and kinetics together with a detailed analysis of a line shape, we have recently shown that we can provide time-resolved measurements of a rapidly compressed plasma in an imploding target [1].

In this paper we discuss bringing together spectroscopic techniques with the goal of measuring plasma gradients. This requires high resolution of the line shape, the development of specialized monochromatic instruments, as well as new algorithms for reducing the data. The challenge is to develop spectroscopic 
analysis that is robust enough to determine the temporally-evolving temperature and density gradients without the use of hydrodynamic calculations. This is possible because we can use the results of multiple spectroscopic techniques and impose a constraint in the fitting routine that requires a self-consistent fit of spatially-averaged and spatially-resolved data. Gradients measured in this way can then be compared to simulations that predict target implosions.

In a previous proceedings of this conference we discussed the competing factors such as the experimental resolution and uncertainties in atomic data that impact line shape measurements in laser-produced plasmas [2]. Here, we will reexamine the argon implosions and discuss the emissivity of the line shape in order to better diagnose plasmas formed in the implosion core of an inertial confinement fusion target.

\section{PLASMA CONDITIONS IN IMPLOSIONS}

In fusion experiments, an imploding capsule emits radiation during its compression. In a typical experiment, highly-ionized atoms are in emission and multiple ionization states are present. Thus, the emission from the plasmas is in the $x$-ray regime. Unlike line shapes from neutral species, these line shapes are primarily due to a single transition with underlying satellite lines, and not to different rotational or vibrational states of an atom. The emitted X-rays are analyzed to determine the density and temperature of the compressed plasma. Time-dependent data is critical to diagnosing the plasma since the implosion takes place in a nanosecond time frame. Many diagnostics can be fielded on implosion experiments. For instance, neutron diagnostics can monitor ignition while pinhole imaging can give information about the density. However, here we focus on the spectral line shape.

An important reason for focusing our efforts on these plasmas is that once the plasma gradients are measured, the compressed plasma can become a testbed to advance line shape theory. These plasmas are of interest because they attain extreme temperature and density regimes. An example of $\rho$-T trajectories of the main fuel in laser-produced implosions is shown in Fig. 1. It is similar to the plot in by Van Horn [3] that shows the regimes of astrophysical importance for hydrogen. In laser fusion, the fuel is composed of deuterium and tritium. These experiments are typically performed with a gas fill of 50 atmospheres of deuterium. As in the Van Horn article. we show two curves that delineate the regimes of interest to these plasmas. The dotted-dashed curve shows where the Fermi energy equals the thermal energy in the plasma. To the left of the curve plasmas are degenerate. The dashed curve delineates where the strong coupling parameter, $\Gamma$, equals 1 . This parameter is defined as the ratio of the Coulombic energy to the thermal energy and to the left of the curve, $\Gamma>1$, meaning the Coulombic energy dominates.

The two solid lines are trajectories of typical laser-produced implosions that were produced on the Nova laser. The thick solid line represents a trajectory for a 1 -ns square laser pulse [4]. The thin sold line is a curve for a shaped 1.7 ns laser drive often used in high convergence experiments similar to those described by Landen et al.[5]. Pulse shaping is used to drive an implosion with a more isentropic and thus more efficient compression. The luel in Nova capsules starts as a gas and moves left to right along the trajectory until the capsule achieves 
peak compression. After that point it cools and the fuel moves along the trajectory to the left, back to lower temperatures. Both of these implosions have been produced at the Nova laser facility at Lawrence Livermore National Laboratory and show the range of compression we can achieve with this laser.

FIGURE 1. Trajectories of the fuel in an imploding capsule. The solid lines

\section{$\log (\mathrm{T})(\mathrm{eV})$}

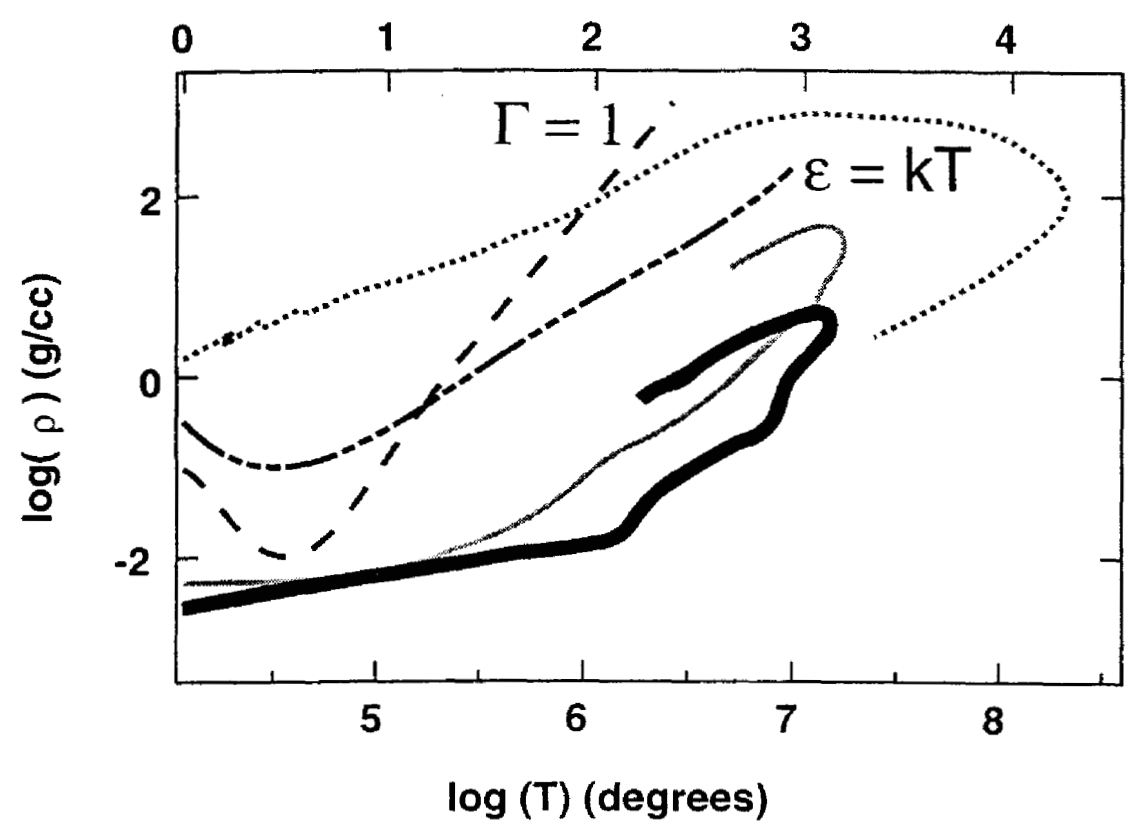

represent implosions that have been performed on the Nova laser. The dashed line is for $\Gamma=1$ and the dotted-dashed line is for $\varepsilon=k T$. The dotted line is a prediction for a fuel in future experiments on the National Ignition Facility.

For comparison, the dotted trajectory is predicted for the future National Ignition Facility, NIF [6]. For NIF experiments cryogenic target capability is planned so the fuel can start at solid density. The NIF capsule spends a significant portion of its time Fermi degenerate. Remaining degenerate is advantageous because it helps increase the compression. Thus the resulting NIF trajectory is qualitatively different from the Nova trajectories because after the capsule achieves peak compression it can then achieve burn. Burn is graphically visible on the $\rho-T$ plot where the fuel remains at high density after peak compression and its trajectory moves to the right as it shoots up in temperature. At later time the fuel cools and the trajectory drops in both density and temperature. The temporal duration of these experiments is implicit in the trajectories; the fuel begins at the leftmost. lowest temperature point of the trajectory and generally moves diagonally to the upper right. The full trajectory represents $\sim 2-4 \mathrm{~ns}$ for the Nova experiments and $\sim 20 \mathrm{~ns}$ for NIF trajectories.

These exotic conditions can produce plasma effects that are difficult to produce by other laboratory methods. One example is the effect of ion dynamics 
on the line widths. These can be predicted by line shape theories, but measurements of the effect have been hampered by plasma gradients.

\section{IMPLOSION EXPERIMENTS}

The baseline experiments on implosions using a 1-ns Nova laser drive have been reported in previous papers [1,7]. These have discussed various aspects of the experiments such as the spectroscopic diagnostic development, temporal temperature and density measurements, and line shape measurements. In this section we emphasize the control on experimental details that lead to highly reproducible data. Furthermore since the initial conditions of targets and irradiation conditions are easily controlled, it possible to perform a systematic study of plasma effects.

The targets consist of two parts: the hohlraum and the capsule. A schematic is shown in figure 2. The hohlraum is a cylindrical Au can that is $25 \mu \mathrm{m}$ thick, $1.6 \mathrm{~mm}$ in diameter, and $2.55 \mathrm{~mm}$ long. It converts laser energy that is deposited on the inside walls to $x$-rays, which then heat the capsule. The hohlraum concept has been the subject of many articles that have explored different geometries and scaling laws that describe the heating $[8,9,10]$. In these experiments, the hohlraum is heated by a constant flux laser pulse and therefore the radiation temperature increases as a function of time. The radiation field is measured for each experiment and is often described by a single number, its effective blackbody temperature, $T_{\mathrm{r}}$. In these experiments, approximately $20 \mathrm{~kJ}$ of laser energy is delivered in a 1-ns flat-top pulse shape at a wavelength of $0.35 \mu \mathrm{m}$ through holes in the endcaps to produce a nearly Planckian radiation drive. A peak $T_{r}$ of $\sim 210 \mathrm{eV}$ is observed at the end of the 1 ns drive. The single parameter $\mathrm{T}_{\mathrm{r}}$ is useful to characterize the temporal energy deposition.

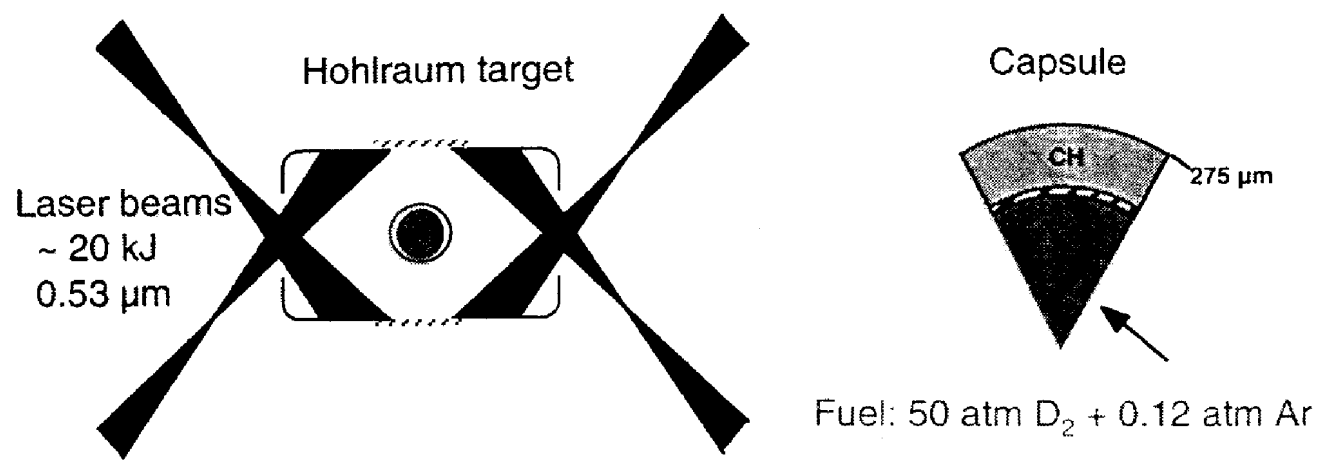

FIGURE 2. Schematic showing the hohlraum target with a capsule inside. Laser beams enter through holes in the endcaps of the Au cylinder and create a radiation drive that implodes the capsule. A wedge cross-section of the capsule is expanded to show the target fuel. 
The capsule itself is composed of a $\mathrm{CH}$ shell filled with gas that has been diffused inside. The shell has a $550 \mu \mathrm{m}$ outer diameter and a $440 \mu \mathrm{m}$ inner diameter. Figure 2 shows a wedge-shaped slice of the spherical capsule with its different layers identified. Capsule fabrication is tightly controlled because defect-free capsules are necessary for producing symmetric implosions. The capsule-to-capsule radial variation is typically kept less than 5\% and can be even more stringent if necessary. The typical tolerances for the $\mathrm{CH}$ shell itself are \pm 20 $\mu \mathrm{m}$ on the inner diameter and $2-3 \mu \mathrm{m}$ in the centering of the gas-filled cavity.

Micro-machining and precision assembly are used to insert the capsule into the center of the hohlraum. First the cylindrical hohlraum is physically cut in half, perpendicular to its axis. Then the capsule is sandwiched between two $\mathrm{CH}$ membranes and suspended in the center of the inner side of the split hohlraum. The other half is then repositioned and the hohlraum is glued back together with the capsule centered at the mid-plane. The thin seam where the $\mathrm{CH}$ membrane is located is visible but does not cause an observable impact on the capsule implosion. The radial and axial centering with respect to the hohlraum body can both be controlled to within $25 \mu \mathrm{m}$ by this fabrication method.

The implosion dynamics can generally be described by a rocket equation where the outside shell of the capsule acts as an ablator and the ablation pressure propels the shell inward [9 and references therein]. This also launches a shock that propagates radially inwards. At the center, the shell converges and the kinetic energy is converted into thermal energy. For most inertial confinement fusion schemes, burn is ideally initiated in a hot spot at the center. Therefore, the most efficient type of implosion keeps the fuel on as low an isentrope as possible in order to minimize heating of the fuel and maximize compression. At stagnation, or maximum compression, the capsule is isobaric.

\section{X-RAY SPECTROSCOPY}

The capsules for these experiments are filled with 50 atmospheres of deuterium and 0.1 atmospheres of Ar. The Ar serves as a dopant to provide spectral lines that can be diagnosed by time-dependent spectroscopy and imaging. The optical depth of a line, $\tau_{o}$, at line center, is given by,

$$
\tau_{\mathrm{o}}=F_{H e} F_{A r} n_{e} R_{f}\left[\left(\pi e^{2} / m c\right) f_{l u} \phi_{o}\right]
$$

where $F_{A r}$ is the Ar number by ion fraction, $F_{H e}$ is the fraction of Ar ions in the He-like ionization state (approximately 0.5 ), $n_{e} R_{f}$ is the peak areal mass density, and $\phi_{o}$ is the emission profile at line center. The quantity in the square brackets is the peak absorption cross-section at line center and its value is $2.3 \times 10^{-19} \mathrm{~cm}^{2}$ for Ar He $\beta$ and $2.9 \times 10^{-19} \mathrm{~cm}^{2}$ for Ar Ly $\beta$. The optical depth is approximately 0.2 at peak compression when the peak areal mass density, measured from secondary $D$ D neutron yield, is $\sim 5 \mathrm{mg} / \mathrm{cm}^{2}$. Hydrodynamic simulations indicate that the implosion dynamics are not significantly altered by varying the Ar concentration from 0.0 to 0.2 atomic-\%. Therefore, in these experiments the dopant is a nonperturbative diagnostic. 


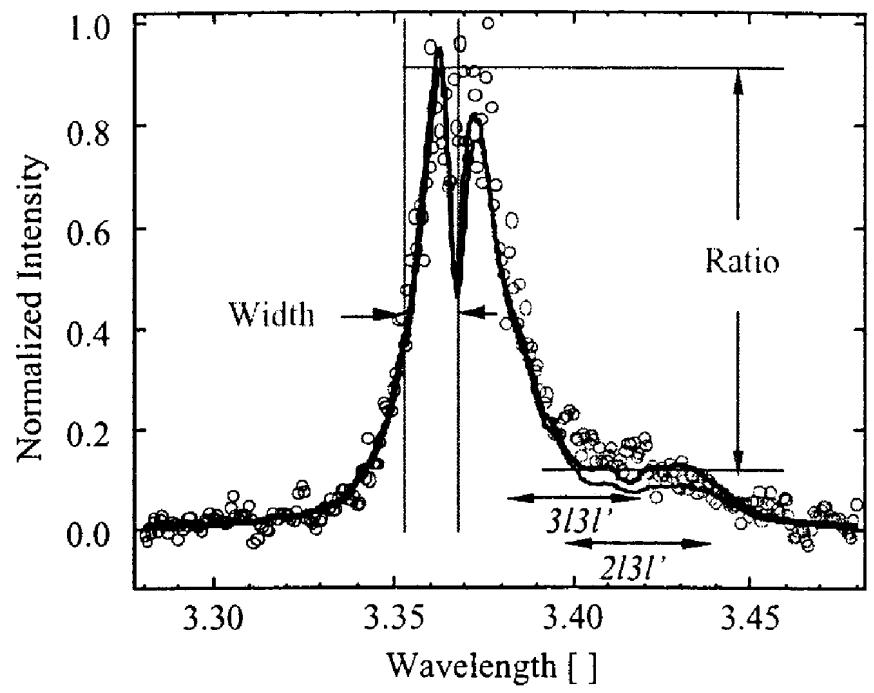

FIGURE 3. Experimental data of the He $\beta$ line (shown by circles) compared to calculations (solid line). Both the line width and the ratio of the $\mathrm{He} \beta$ to its satellites are used to diagnose core-averaged temperatures and densities.

The spectroscopic instruments are low and high resolution spectrometers which record the Ar K-shell emission. The paper by Woolsey et al. [7] gives detail on the full complement of diagnostics and data analysis. For convenience, we repeat the spectroscopic details important to the foundation for these studies. An example of the data from the flat RAP Bragg crystal that was coupled to an $x$-ray streak camera is shown in figure 3 . It was aligned for a spectral range of $2.7 \AA$ to $4.2 \AA$ with a spectral resolving power of 500 over the Ar K-shell $n=1-3$ transitions lines of interest. The same line shape was also obtained with a spectral resolution of 1800 . On the figure the line width and ratio which were used for ne and $\mathrm{T}_{\mathrm{e}}$ diagnostics are indicated.

The reduced data for temperature and density measurements are shown in figure 4 . On the left, the $\mathrm{n}_{\mathrm{e}}$ time histories from five implosion experiments are plotted where each symbol represents a different experiment. Also shown is the result from the HYADES simulation [10] of the half-mass point $n_{\mathrm{e}}$ time history represented by the solid line curve. The code was run with an ideal gas equation of state and non-LTE average atom physics to model the fuel. The $n_{e}$ are extracted by measuring the half-width at half-maximum (HWHM) of the blue side of the He $\beta$. The core diameter measured by $x$-ray pinhole imaging was $\sim 50 \mu \mathrm{m}$, which represents a compression of approximately 8 from its initial diameter.

The temperature measurements are also shown in figure 4 and are derived from fitting the $\mathrm{He} \beta$ resonance line and its Li-like satellites on the long wavelength side. Over 1.4 to $1.7 \mathrm{~ns}$ after the start of the laser pulse, the temperatures range from 800 to $1000 \mathrm{eV}$ with a much wider spread than the electron density measurements. Here the 1-D hydrodynamic simulation provides 

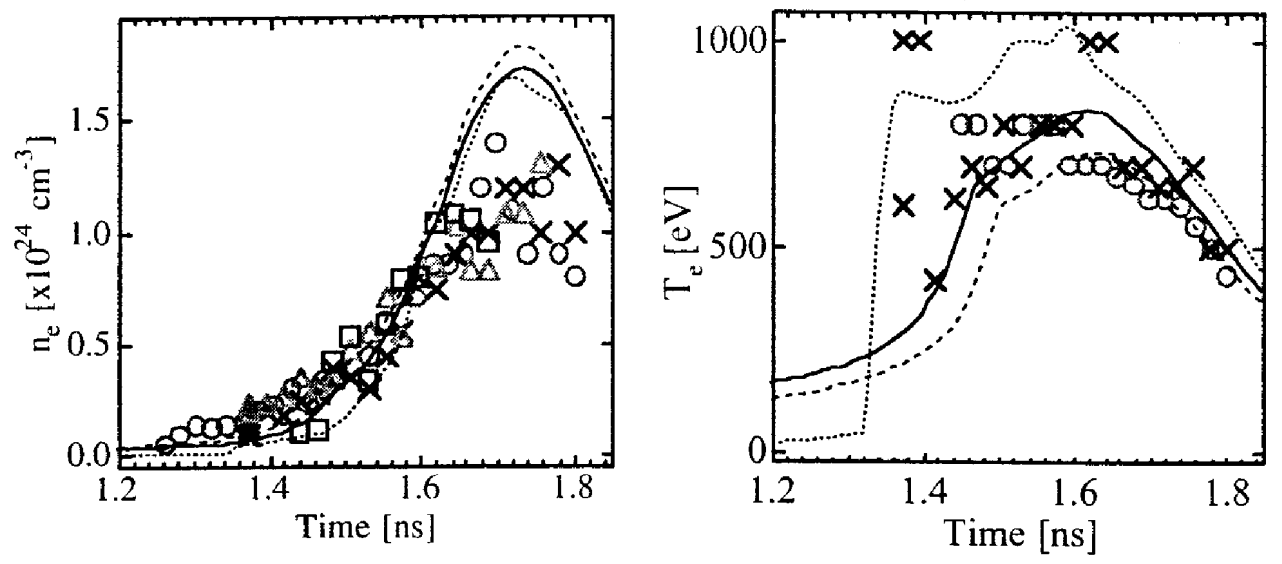

FIGURE 4. The measured $n_{e}$ and $T_{e}$ compared to l-D HYADES calculations. The solid line represents the $50 \%$ mass point, the dotted lines show the $30 \%$ mass point and the fuel-CH interface.

insight into the measured temperature spread because it reveals a significant temperature gradient in the fuel. The time histories of three zones in the imploding $\mathrm{D}_{2} / \mathrm{Ar}$ simulations are overplotted in the figure: a point at the inner $30 \%$ of the mass, the $50 \%$ mass point, and the outer zone point near the $\mathrm{D}_{2} / \mathrm{Ar}$ $\mathrm{CH}$ interface. The three curves show that the simulations predict a large temperature gradient early in the implosion with more modest gradients at the peak density.

In summary, these experiments provide high quality time-resolved spectroscopic data. For ICF, the core-averaged $\mathrm{T}_{\mathrm{e}}$ and $\mathrm{N}_{\mathrm{e}}$ measurements have helped tune the design of inertial confinement fusion targets to improve the implosion.

\section{SPATIALLY-RESOLVED PLASMA PARAMETERS}

However, as noted before, burn of the fuel initiates at the center of the capsule. One-dimensional calculations of the 1-ns laser pulse case predict a temperature gradient, which peaks at the center where the fusion process is initiated. Unfortunately, due to electron broadening and changing satellite contributions underneath the primary $n=3-1$ transition, the spatially-averaged line shape is not sensitive to different gradients. To illustrate the effect of the gradient, figure 5 shows three pairs of $\mathrm{n}_{\mathrm{e}}$ and $\mathrm{T}_{\mathrm{e}}$ gradients as a function of spatial zones [11]. Zone zero corresponds to the center of the imploding capsule. For three idealized temperature-density combinations, the spatially-averaged line shapes are virtually the same. Therefore, to more closely understand and tune implosion dynamics, this core-averaged data is inadequate. 

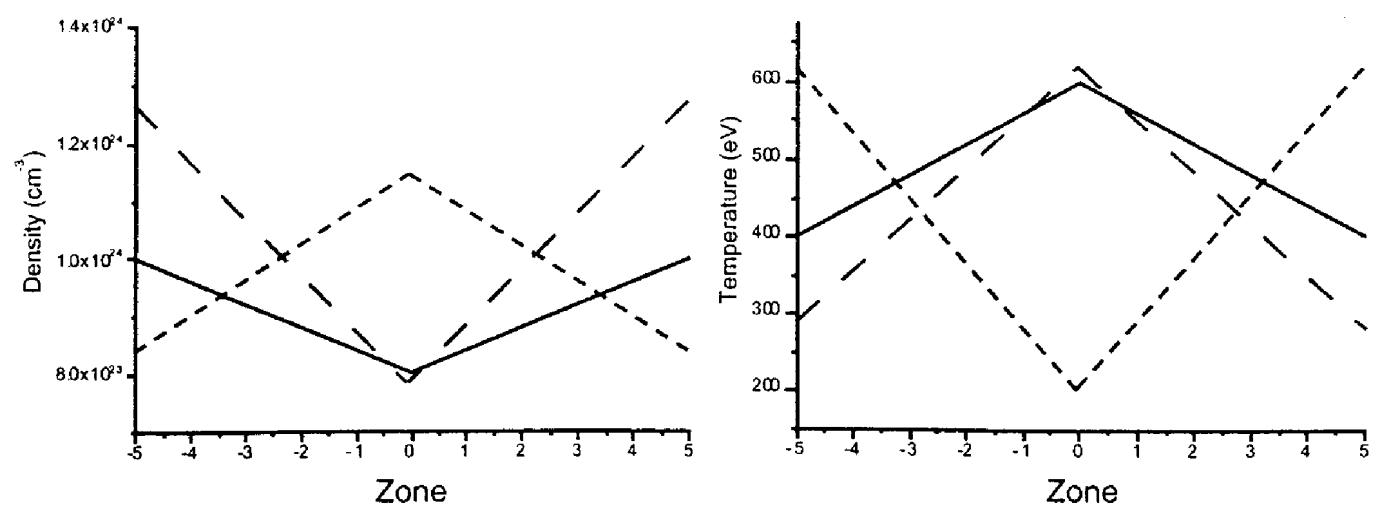

FIGURE 5. Sample temperature and density profiles used to assess the sensitivity of the line shape and the emissivity to plasma gradients. The line shape is calculated for the pair of $n_{e}$ and $T_{e}$ given by the solid, short dashed or long dashed gradients.

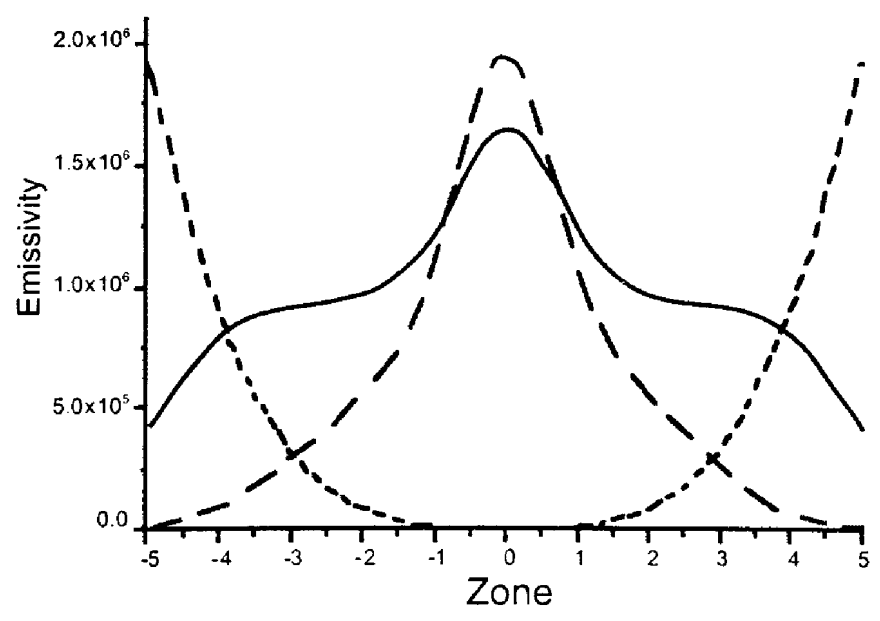

FIGURE 6. Emissivity calculated for the different gradients shown in figure 5.

However, it is possible to sample a frequency range of the line shape that is narrower than the full line shape. We find that for a judicious selection on the blue wing where there are fewer satellite contributions, calculations of the emissivity using the same gradients are not the same. Figure 6 clearly shows that the emissivity profiles can differentate between the plasma gradients.

The most straightforward way to obtain spatially-resolved data is to field a streaked $x$-ray pinhole camera. This provides data which is spatially-resolved in one dimension and temporally-resolved in the other dimension. However, the bandpass of this instrument is fairly broad since it is determined by cold K-edge filters. A more sophisticated instrument would be a spatially-resolving spectrometer. For both types of instruments, the data from imploding cores must 
be Abel inverted to obtain emissivity as a function of the radius of the core. However, to compensate for the reflectivities of Bragg crystals, the source must be sufficiently bright to be successful with a spectrometer.

We have performed a sensitivity check to determine the feasibility and necessity of using a spatially-resolving spectrometer [12]. In these tests we compared data from both kinds of instruments. Abel inversions of monochromatic data show radial emissivity peaked at the center for the monochromatic images of the He $\beta$ line taken near the peak of the implosion at 1.5 ns and $1.7 \mathrm{~ns}$. However, the K-shell emission from the streaked pinhole camera produces an emissivity that peaks at the outer radius. Thus for self-consistent fits of averaged data involving the $\mathrm{He} \beta$ line, the Abel inversion of the group of $\mathrm{K}$ shell lines, will not be sufficiently accurate for detailed cross-correlations. These results emphasize the need for a high resolution monochromatic instrument that is tailored for a narrow spectral bandpass.

Therefore, we are currently developing a new diagnostic to obtain spatiallyresolved core emission at high magnification. Since the He $\beta$ line is typically 30 $\mathrm{m} \AA$ in width, it is wide enough to use Bragg crystals to obtain monochromatic images of the blue side of the line, free from satellite emission. Our goal is to obtain emission as a function of space, $x$, over a bandwidth of $10 \mathrm{~m} \AA$.

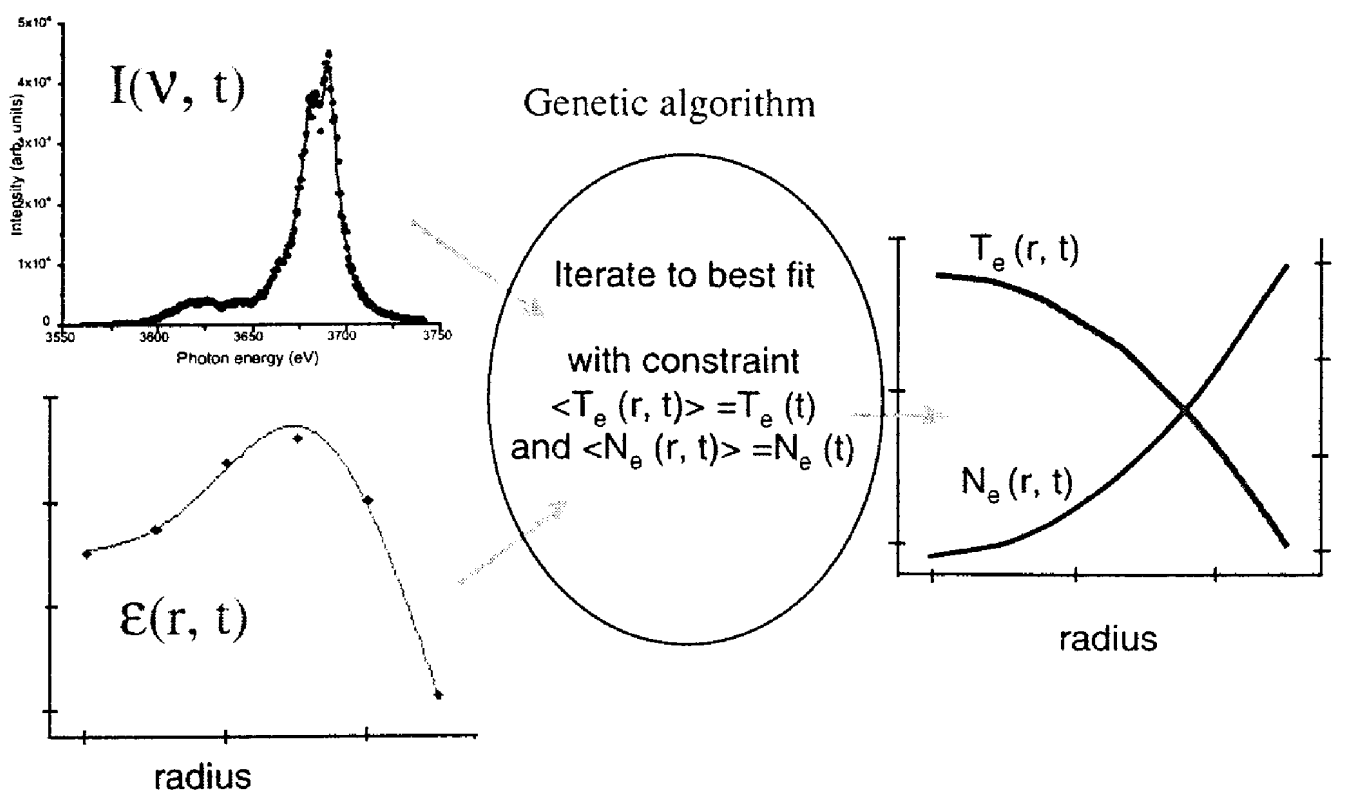

FIGURE 7. Schematic of the genetic search algorithm that solves for the temperature and density gradients for a given set of core-averaged data and emissivity data. 
Finally, however, even with spatially-resolved data, it is still a challenge to determine the plasma gradients. Analysis requires that the radiation transport equation be solved self-consistently with the kinetics equations for the emitting ion level populations. In order to solve this problem, we have turned to artificial intelligence search algorithms [13,14]. For unately, the field of evolutionary computations has developed genetic algorithms, which are based on Darwin's evolutionary theory. These algorithms are well-suited to solve problems whose solutions are difficult to find, but easy to check. To implement this algorithm, the first step is to define a search space of solutions. Then we choose the criteria for the fit and look for the solutions by finding an extremum in the search space. Initially only a single criterion could determine the fit. However, the development of "Niched Pareto" genetic algorithms expanded their application to problems that could be solved by finding a family of solutions which are subject to multiple criteria. Consequently, the advances in evolutionary computations are now being applied domains outside of biology.

In our problem of finding self-consistent gradients, we define a search space of $\mathrm{T}_{\mathrm{e}}(\mathrm{r})$ and $\mathrm{N}_{\mathrm{e}}(\mathrm{r})$ subject to the constraint that the time averages match the data. The proper weighting for the spatial averages is by the emissivity. The criteria for the fit are that we match the core-averaged $I(v)$ and the emissivity $\varepsilon(r)$. We then examine the set of solutions to maximize the fitness of the solution. The uniqueness of the solution is determined by its convergence to the space-averaged temperature and density. This process is schematically illustrated in figure 7. This spatially-resolved data, coupled with core-averaged measurements, can then be used to determine a self-consistent radial profile of the plasma gradients at each instant in time.

\section{CONCLUSIONS}

Laser-driven implosion experiments produce unique plasmas at high energy densities. Experimental techniques and target fabrication are well-developed and produce extremely reproducible high quality data. Previous experiments have shown that we can use purely spectroscopic techniques to diagnose the temperature and density of an imploding core.

The next step is to measure the plasma gradients. This requires a new instrument that provides spatially-resolved monochromatic images. This data can be Abel inverted to provide emissivity as a function of radius in the core. Then by using spatially-resolved and spatially-averaged spectroscopic data, genetic algorithms can be used to solve for the gradients by requiring self-consistent fits. Work is now underway to build an imaging spectrograph that has a bandpass of $\sim 10 \mathrm{~mA}$ to measure the $\mathrm{He} \beta$ emissivity.

In summary, measurements of the gradients can be used to test the accuracy of large-scale integrated calculations. As experiments have gotten more sophisticated, we can move beyond just diagnosing plasma temperature and density. Now we can actually start to use spectral line shapes to benchmark the hydrodynamic calculations. Moreover, diagnosing gradients moves us a step closer to turning implosion plasmas into testbeds for line shape theory. 


\section{ACKNOWLEDGEMENTS}

This work was performed under the auspices of the U.S. Department of Energy by the University of California Lawrence Livermore National Laboratory under Contract No. W-7405-ENG-48. This work was also supported in part by DOE HEDS grant number DE-FG03-98DP00213 to Howard University.

\section{REFERENCES}

1.Woolsey, N. C. , Hammel, B. A., Keane, C. J. , et al., J. Quant. Spect. Radiat. Transfer 58, 975989 (1997).

2. Back, C. A., Woolsey, N. C., Asfaw, A., et al., "Experiments on Hot and Dense Laser-Produced Plasmas" in Spectral Line Shapes, edited by M. Zoppi, AIP Conference Proceedings 386, New York, 1997, pp. 35-44.

3. Van Horn, H., Science 252, 384-389 (1991).

4. Kauffman, R. L. et al., Phys. Rev. Lett. 73, 2320-2323 (1994).

5. Landen, O. L., et al., Bull. Am. Phys. Soc. 39, 1686 (1994).

6. private communication, T. Dittrich, 1998.

7. Woolsey, N. C. , et al. Phys. Rev. E 53, 6396-402 (1996) ; Woolsey, N. C. et al. Phys. Rev. E 56, 2314-17 (1997).

8. Kaiser, N. Meyer-ter-Vehn, J., and Sigel, R. Phys. Fluids B, 1, 1747-52 (1989); Eidmann, K., Schmalz, R. F., and Sigel, R., Phys. Fluids B.2 208-17 (1990).

9. Lindl, J., Phys. Plasmas 2, 3933- 4024 (1995).

10. Larsen, J. T., and Lane, S., J. Quant. Spectrosc. Radiat. Transfer 51, 179-86 (1994).

11. Private communication, I. Golovkin, 1999.

12. Back, C. A., Woolsey, N. C., Missalla, T., et al. Ap. J. Supp. 127, 227-232 (2000).

13. Horn, J. Nafpliotis, N., "Multiobjective Optimization Using the Niched Pareto Genetic Algorithm" Illinois Genetic Algorithms Laboratory report No 93005, Urbana-Champaign, 1993, pp. 1-32 also published in part in Proceedings of the first IEEE conference on Evolutionary Computation, IEEE vol. 1 (1994).

14. Golovkin, I., Mancini, R., Louis, S., et al., " Plasma X-Ray Spectra Analysis Using Genetic Algorithms" in Proceedings of the Genetic and Evolutionary Computation Conference, San Francisco, 1999, pp. 1529-34. 